\title{
The Impact of Epidural Analgesia on Postoperative Outcome After Major Abdominal Surgery
}

\author{
Iulia Cindea, Alina Balcan, Viorel Gherghina, Bianca Samoila, \\ Dan Costea, Catalin Grasa and Gheorghe Nicolae \\ Emergency Clinical Hospital of Constantza, \\ Romania
}

\section{Introduction}

Despite the spread of minimally invasive surgical techniques and improvements in postoperative management, major abdominal procedures still induce neurohumoral changes responsible for postoperative pain, various organ dysfunctions, prolonged hospitalization and convalescence.

One of the main concerns of patients presenting for major abdominal surgery and anesthesiologists involved in postoperative care is postoperative analgesia. The most common postoperative pain control methods are epidural infusion of a combination of local anesthetic and an opioid or local anesthetic alone and administration of intravenous opiates, both of them being accepted options following major abdominal procedures.

Parenteral opioids can provide adequate analgesia but pain on movement is generally less controlled. Their side-effects, in particular respiratory depression, limit the dose that can be given, therefore resulting in suboptimal analgesia for some subjects.

Epidural analgesia confers excellent pain relief and complete dynamic analgesia leading to a substantial reduction in the surgical stress response. Epidural analgesia seems to provide favorable effects on coagulation and homeostasis, as well as on cardio-respiratory, gastrointestinal and immune functions, all these potential positive influences being theoretically translated into an improved quality of patient recovery.

The outcome of different treatment methods for postoperative pain after major abdominal surgery has been an issue of controversy, mainly because of the increasingly need for large patient numbers in any individual trial in order to derive reliable conclusions. Epidural analgesia is the most investigated strategy, being assumed that it has the greatest theoretical potential to considerably modify postoperative outcome.

This chapter systematically reviews a large-scale data to acquire suitable evidence on the effects of epidural analgesia on postoperative outcome, contributing to the collaborative efforts of anesthesiologists and surgeons for the improvement of patient recovery after major abdominal surgery. 


\section{Method for systematic review}

A broad search strategy including Medline and Cochrane Library databases has been conducted. Meta-analyses and large controlled trials that randomized major abdominal surgery patients to either epidural or opioid-based systemic analgesia and that are reported on postoperative outcomes have been retrieved. All references within relevant papers have been further checked for additional studies.

The above mentioned data sources have been searched from January 1990 to December 2010, our investigation being limited to the last two decades because of progress of surgical techniques, improvement in quality of postoperative care and implementation of rational use of aggressive pain control.

The following search terms have been used in various combinations: major abdominal surgery, postoperative pain, postoperative analgesia, epidural analgesia, patient-controlled analgesia, postoperative complications, pulmonary complications, respiratory insufficiency, pneumonia, myocardial ischemia, myocardial infarction, heart failure, dysrhythmias, ileus, infection, surgical wound infection, deep venous thrombosis, cognitive disorders, postoperative mortality, cost-efficiency ratio, postoperative stress response, risks of epidural analgesia, duration of hospital stay.

Firstly, the trials have been screened per inclusion and exclusion criteria. Secondly, all the inclusion criteria have been validated for the accepted studies before the data extraction process. In those cases in which uncertainty arose, the articles to be excluded have been validated, too.

According to the study design, the selected trials have been stratified into levels of evidence ranged from I to IV where level I: systematic review of randomized controlled trials, level II: randomized controlled trials, level III: non-randomized controlled trials or from cohort or case-control analytical studies and level IV: expert opinion.

Trials in adult patients in which epidural analgesia has been started immediately postoperatively and has been maintained for 3 days after surgery, have been included. The included studies have tested thoracic or lumbar epidurals, the analgesic regimen consisting of local anesthetic alone or a combination of local anesthetic and opioids. Controls have received subcutaneous, intravenous or intramuscular opioids that have been given on demand, regularly or via patient-controlled analgesia.

Considering that best evidence is coming from large patient numbers, preference has been given to the large sample sizes. Consequently, we have chosen an $n \geq 100$ (100 individuals per randomized group) as suitable inclusion criterion for individual studies. This option is based on highest reported incidences of postoperative morbidity to maximize capture of randomized controlled trials.

We have noted the type of epidural analgesia involving the level of catheter insertion, duration and analgesic regimen. For systemic analgesia, the route of administration and the regimen have been recorded, too. Definitions of postoperative outcome parameters have been taken as reported in the original articles.

Those trials in which controls have received epidural analgesia or have not received systemic opioids have been rejected. Trials with fewer than 100 patients per group have 
been excluded, too. Studies which data on postoperative outcome assessment could not be extracted have been considered out of the interest of this analysis.

Based on these criteria we have selected the eligible meta-analyses, systematic reviews, randomized controlled trials and observational database articles to gather evidence on the impact of epidural analgesia on postoperative outcome in major abdominal surgery patients.

\section{Effects of epidural analgesia on major postoperative outcomes}

\subsection{Effect of epidural analgesia on pulmonary function}

Postoperative pulmonary dysfunction after major abdominal surgery has a multifactorial pathophysiology including deranged central ventilatory control, alteration of normal respiratory muscle activity, diaphragmatic dysfunction, consequence of a reflex inhibition of phrenic nerve activity and postoperative pain that contributes to the impairment of pulmonary mechanics (Liu \& Wu, 2007; Nimmo, 2004). From far, the most important effect of major abdominal surgery on pulmonary function is the reduction of functional residual capacity (FRC) determined by diaphragmatic dysfunction, pain-related reduction of spontaneous ventilation and decreased chest wall compliance (Moraca et al., 2003; Warner, 2000).

Postoperative pulmonary complications, as common as cardiac complications in the context of major abdominal surgery, may prolong the duration of hospitalization and carry an increased risk of mortality. Epidural analgesia confers optimal postoperative pain control thus resulting in pain-free ventilation, coughing and better cooperation with physiotherapy (Wu et al., 2005).

On the other hand, the superior pain relief provided by this technique reduces the consumption of parenteral opioids that adversely affect ventilation and gas exchange (Nimmo, 2004).

The interruption of the reflex inhibition of phrenic nerve by segmental block is likely to have a beneficial effect, mainly by allowing normal diaphragm function, thus improving diaphragmatic activity (Liu \& Wu, 2007; Nimmo, 2004).

Epidural analgesia obtunds the stress response to major surgery, fact that is of particular interest for the clinician, since it reduces the level of postoperative immunosuppression, thus contributing to decrease of pulmonary infections incidence (Nimmo, 2004).

Taking into account that the effects on respiratory muscle function are complex, it has been suggested that epidural analgesia for pulmonary function might be unfavorable during early postoperative period due to potential paralysis of intercostals or abdominals and changes in bronchial tone (Liu \& Wu, 2007; Waurick \& Van Aken, 2005). However, it has been demonstrated that epidural analgesia does not impair respiratory muscle strength or airway flow even in patients with end-stage chronic obstructive pulmonary disease, resulting, by contrast, in better ventilatory mechanics and optimal respiratory muscle force generation (Groeben et al., 2002; Gruber et al., 2001; Waurick \& Van Aken, 2005).

Even the biological basis underlying these associations is unclear, it is well documented that epidural analgesia improves some measurable pulmonary function parameters such as tidal volume, vital capacity, forced vital capacity at 24 hours, forced expiratory volume in 1 
second at 24 hours, peak expiratory flow rate at 24 hours. It has been noted a significantly increased arterial oxygen pressure at 24 and 72 hours, too (Moraca et al., 2003; Pöpping et al., 2008).

However, it should be noted that, there is no documented significant correlation between postoperative pulmonary function tests and the incidence of pulmonary complications (Ballantyne et al., 1998; Kehlet \& Holte, 2001; Moraca et al., 2003).

Therefore, such surrogate outcome measures seem to be inappropriate for the investigation of postoperative pulmonary morbidity. Other markers, namely the incidence of pneumonia respiratory failure, atelectasis and hypoxemia have been demonstrated to be more adequate in this respect (Kehlet \& Holte, 2001; Moraca et al., 2003).

Thus, large meta-analyses of randomized controlled trials pointed out a significant reduction in pneumonia, as well as in respiratory depression in major abdominal surgery subjects treated postoperatively with epidural analgesia, compared with those receiving systemic analgesia (PCA) (Liu \& Wu, 2007; Moraca et al., 2003). This finding is in agreement with other studies that noted a significantly decreased risk of respiratory failure associated with epidural analgesia for patients undergoing major abdominal procedures ( Jiang et al., 2005; Park et al., 2001; Rigg et al., 2002; Werawatganon \& Charuluxananan, 2005).

An earlier meta-analysis on randomized clinical trials examining the use of epidural analgesia in major abdominal surgery concluded that this technique significantly reduces the incidence of pulmonary complications overall, being specifically associated with significantly less atelectasis and pneumonia (Ballantyne et al., 1998; Moraca et al., 2003).

Over the last decade, the protective effect of epidural analgesia on postoperative pulmonary complications has decreased, compared with conventional systemic analgesia. This phenomenon does not seem to be related to a decrease in the efficacy of the epidurals per se, but rather to a reduction in baseline risk of such complications. Modification of standard care in patients undergoing major abdominal procedures including respiratory physiotherapy, the routine use of naso-gastric tube, prophylactic antibiotics and early mobilization, may all have favorably influenced the risk of pulmonary complications (Pöpping et al., 2008).

In conclusion, consistent data from meta-analyses and large randomized controlled trials support the potential physiologic benefit of epidural analgesia for reducing postoperative pulmonary complications in major abdominal surgery patients.

\subsection{Effect of epidural analgesia on postoperative pain}

Adequate postoperative pain control is a key therapeutic component of the complex multimodal rehabilitation program after major abdominal procedures (Bartha et al., 2006). In this context, epidural technique is considered by many the standard-care strategy, because it provides complete analgesia and especially effective dynamic pain relief. Such postoperative benefits allow the subject to early mobilize and resume normal activities (Block et al., 2003; Dolin et al., 2002). The optimal level of postoperative analgesia is usually achieved with a combination of epidural local anesthetic and opioid (Kehlet, 1994, 1997; Nimmo, 2004; Wheatley et al., 2001). 
The comparison of epidural therapy versus systemic opioids after major abdominal surgery, that has been studied in many individual trials and has been reviewed in large metaanalyses, has pointed out that the epidural technique provided a significantly better analgesia at all time points up to the third postoperative day (Waurick \& Van Aken, 2005).

Large-scale observational data acquired from systematic reviews comprising large number of patients have noted that postoperative epidural analgesia was associated with significantly lower incidence of severe and moderate pain by comparison to systemic opioids strategy, after major abdominal procedures (Waurick \& Van Aken, 2005).

"Preemptive analgesia" is an attractive concept revealing that blockade of nociception by epidural analgesia before surgical manipulation will positively affect the perception of subsequent painful stimuli. Thus, pain perception and overall analgesic needs could significantly decrease during hospitalization and the incidence of long-term pain after major abdominal procedures might be reduced in patients treated with preemptive epidural analgesia. Despite encouraging findings from small clinical trials, this effect has not been reproduced in other studies. The validity and clinical relevance of preemptive epidural analgesia is still controversial and more trials investigating the effect of this technique on either acute or chronic pain after major abdominal surgery are warranted (Kehlet \& Dahl, 2003; Moraca et al., 2003).

Clearly, epidural strategy is associated with a statistically significant and clinically relevant improvement of postoperative pain control in patients undergoing major abdominal surgery, although it requires specific technical and pharmacological skills, as well as professional monitoring of the subjects.

\subsection{Effect of epidural analgesia on gastrointestinal function}

Gastrointestinal paralysis, nausea and vomiting recognized as common clinical problems after major abdominal surgery may last for days and prolong hospitalization and convalescence. Although the pathophysiology of postoperative ileus is multifactorial, primary mechanisms include local inflammatory response, nociceptive reflexes, activation of inhibitory splanchnic reflexes and systemically applied opioids, all of these being subject to modification by postoperative epidural analgesia (Kehlet \& Holte, 2001; Liu \& Wu, 2007; Nimmo, 2004; Waurick \& Van Aken, 2005).

After major abdominal procedures, epidural analgesia provides superior pain control by blocking nociceptive reflexes, thus limiting systemic opioids use, a documented factor that increases the risk of ileus ( $\mathrm{Liu} \& \mathrm{Wu}, 2007 ; \mathrm{Wu}$ et al., 2005). The sympathetic block from epidural analgesia may attenuate postoperative reflex inhibition of gastrointestinal motility thus reducing the duration of ileus and allowing early enteral feeding (Bauer \& Boeckxstaens, 2004; Jørgensen et al., 2000; Mythen, 2005). Attenuation of the stress response to major abdominal surgery reduces the inflammatory response that contributes to the maintenance of postoperative ileus (Mythen, 2005; Nimmo, 2004).

There has been debate about whether the ileus-reducing effect associated with postoperative epidural analgesia may pose a risk to the integrity of intestinal anastomoses. Data from published randomized clinical studies and large meta-analyses have consistently indicated that thoracic epidural analgesia, suitable after major abdominal surgery, blocks the activity 
of sympathetic fibres innervating the mesenterial blood vessels, thus improving the mucosal blood flow, even under circumstances of reduced perfusion pressure. In this way, postoperative epidural analgesia could contribute to healing rather than to anastomotic dehiscence when compared to systemic opioid analgesia (Kehlet \& Holte, 2001; Liu \& Wu, 2007; Waurick \& Van Aken, 2005).

In summary postoperative epidural analgesia significantly reduces postoperative ileus, with major clinical impact allowing early recovery of gut function, subsequently early enteral nutrition with beneficial effect on postoperative nitrogen balance and total body protein preservation, thus resulting in better outcome.

The great majority of randomized clinical trials investigated have demonstrated a significant reduction in postoperative ileus with epidural technique by comparison to systemic opioid analgesia (Kehlet \& Holte, 2001; Waurick \& Van Aken, 2005).

\subsection{Effect of epidural analgesia on stress response to major abdominal surgery}

The postoperative stress response to major abdominal surgery is defined as a cascade of effects that result from activation of neural, metabolic and endocrine pathways with initiation of coagulation and inflammatory mechanisms (Nimmo, 2004).

This postoperative surgical stress response could contribute to various organ dysfunctions in susceptible individuals, thus leading to a difficult and prolonged recovery and rehabilitation (Kehlet \& Holte, 2001). There is a common consensus that a reduction in the stress response is followed by a reduced postoperative major morbidity and improved surgical outcome (Holte \& Kehlet, 2002; Kehlet \& Holte, 2001; Nimmo, 2004).

It has been postulated that pain relief represents an effective method to reduce surgical stress response, since afferent neural stimuli and activation of autonomic nervous system together with other reflexes by pain serve as a major release mechanism of the endocrine and metabolic responses (Kehlet \& Holte, 2001). Thus, one of the beneficial effects of epidural analgesia results from obtunding the postoperative stress response by provision of optimal analgesia.

However, the efficacy in modulating endocrine-metabolic response substantially depends on the level of epidural blockade, its duration, the analgesic regimen (local anestheticbased regimen or local anesthetic-opioid combination regimen), as well as the administration technique (continuous epidural analgesia or patient-controlled postoperative epidural analgesia) and the literature has been confounded by high heterogeneity in this respect. Thus, many reported randomized studies with different analgesia regimens have been combined in meta-analyses, furthermore often there is no distinction between thoracic and lumbar epidural blockade or various techniques of administration, facts that limit the interpretation of these findings (Grass, 2000; Kehlet \& Holte, 2001; Wheatley et al., 2001).

However, use of epidural analgesia to reduce the deleterious effects of postoperative stress response has been demonstrated to improve the clinical outcome, becoming a popular strategy in major abdominal surgery patients. 


\subsection{Effect of epidural analgesia on muscle function}

Epidural analgesia is particularly effective at providing complete pain relief at movement (Nimmo, 2004; Wheatley et al., 2001). On the other hand, alteration of postoperative stress response has been shown to reduce postoperative catabolism resulting in less muscle wasting and postoperative weakness (Nimmo, 2004; Rigg et al., 2002; Wheatley et al., 2001).

These effects in conjunction with early postoperative feeding could contribute to muscle strength and an improved exercise tolerance, allowing the patient to sit out of bed, even on the day of surgery and to actively mobilize next day (Holte \& Kehlet, 2002; Nimmo, 2004).

Conclusively, there is general agreement that epidural analgesia has positive effect on postoperative mobilization, faster physical rehabilitation being recognized as a prerequisite for early postoperative recovery after major abdominal surgery.

\subsection{Effect of epidural analgesia on cardiovascular function}

Postoperative cardiovascular complications, a major cause of postoperative death, are typically reported after major abdominal procedures, particularly in elderly that carry a high risk for such events (Liu \& Wu, 2007).

Poorly controlled postoperative pain and stress response lead to sympathetic arousal that increases myocardial oxygen demand by increasing heart rate, arterial blood pressure and contractility (Nygärd et al., 2005). In addition, sympathetic activation could decrease myocardial oxygen supply by enhancing the risk of thromboembolic complications, especially coronary thrombosis (Devereaux et al., 2005; Waurick \& Van Aken, 2005).

Thoracic epidural analgesia reduces sympathetic activity, providing a favorable balance of myocardial oxygen. Thus, thoracic epidural analgesia improves myocardial blood flow by selectively increasing the diameter of stenotic epicardial coronary arteries while maintaining coronary perfusion pressure, and decreases myocardial oxygen demand by reducing pain, heart rate and systemic vascular resistance (Liu \& Wu, 2007; Peyton et al., 2003; Waurick \& Van Aken, 2005).

Procedure-specific meta-analyses and randomized controlled trials have been focused on the role of postoperative epidural analgesia compared to standard intravenous treatment in terms of cardiac protection after major abdominal procedures. Thus, subjects with thoracic epidural analgesia have experienced lower number of ischemic episodes of shorter duration, a significant reduction in the incidence of myocardial infarction, heart failure and dysrhythmias (Beattie et al., 2001; Block et al., 2003; Kehlet \& Holte, 2001; Waurick \& Van Aken, 2005).

Reaching valid conclusions based on these findings could be difficult because of differences in definitions of cardiovascular adverse events, the use of different analgesic techniques and regimens and the inadequately sized studies to analyze cardiovascular complications (Kehlet \& Holte, 2001; Liu \& Wu, 2007).

Regardless the above-mentioned limits, we have found enough evidence to suggest a clinically relevant benefit of epidural analgesia and thoracic epidural analgesia in particular, in reduction of postoperative cardiac morbidity after major abdominal surgery, the largest 
improvement being observed in cardiac high-risk patients (Liu \& Wu, 2007; Waurick \& Van Aken, 2005).

In summary, although the impact on cardiac morbidity continues to demand our attention, the clinically significant advantages of postoperative epidural block after major abdominal procedures have been demonstrated particularly in specific categories of subjects bearing preoperative cardiovascular co-morbidities.

\subsection{Effect of epidural analgesia on coagulation}

The coagulation-fibrinolysis balance is disturbed in association with major abdominal procedures (Liu \& Wu, 2007). Thus, after surgical incision there have been identified high plasma levels of tissue factor, tissue plasminogen activator, plasminogen activator inhibitor-1, von Willebrand factor which lead to a hypercoagulability and hypofibrinolytic state with resultant risk of formation of deep venous thrombosis and potentially fatal pulmonary embolism ( Agnelli, 2004; Bombeli \& Spahn, 2004; Liu \& Wu, 2007; Roderick et al., 2005). Use of effective methods of thromboprophylaxis in surgical practice makes difficult the estimation of current incidences of deep venous thrombosis and pulmonary embolism (Liu \& Wu, 2007).

Epidural analgesia provides potential benefits such as reduction of coagulation proteins and platelet activity, preservation of fibrinolytic function by obtunding the postoperative stress response and increase of arterial and venous blood flow, thus preventing postoperative coagulation-related complications (Kehlet \& Holte, 2001; Liu \& Wu, 2007; Moraca et al., 2003). Early postoperative mobilization facilitated by dynamic analgesia could play a protective role, too (Nimmo, 2004).

However, systematic reviews of randomized clinical trials in major abdominal surgery have indicated a non-significant reduction of thromboembolic complications, assessed by phlebography or iodine-fibrinogen scans, when postoperative epidural analgesic regimen is compared with systemic opioid technique (Kehlet \& Holte, 2001; Liu \& Wu, 2007).

Furthermore, another specific analysis of randomized studies has been noted, beside the extremely low incidences of thromboembolic complications due to thromboprophylaxis, no differences between groups with epidural analgesia and those with systemic opioids, after major abdominal procedures (Kehlet \& Holte, 2001; Liu \& Wu, 2007).

In conclusion, in the era of rapidly evolving strategies of effective thromboprophylaxis in the field of major abdominal surgery, there is minimal evidence that epidural analgesia could attenuate the risk of postoperative coagulation-related complications.

\subsection{Effect of epidural analgesia on immune function}

Major abdominal surgery is associated with an imbalance between proinflammatory and antiinflammatory cytokines and immunocompetent cells in favor of an early hyperinflammatory response (Sido et al., 2004). The immune system impairment determined by extensive tissue damage, anesthesia and uncontrolled postoperative pain, consist of release of tumor necrosis factor $\alpha$, interleukin-1, interleukin-2, interleukin-6, neutrophil activation, polymorphonuclear and macrophage oxidative activity, lead to a significant cell-mediated immunosuppression manifested by monocyte deactivation and phagocytes inhibition (Liu \& Wu, 2007; Yokoyama et al., 2005). 
Epidural analgesia could improve postoperative immune function, at least theoretically, by reducing proinflammatory response as well as lymphocyte suppression (Beilin et al., 2003; Liu \& Wu, 2007; Waurick \& Van Aken, 2005). The studies that have investigated the effect of epidural analgesia compared to systemic opioid regimen on postoperative immune function in major abdominal surgery patients, have noted reduced suppression of lymphocyte proliferation and attenuated proinflammatory response (Beilin et al., 2003; Yokoyama et al., 2005).

Insufficient controlled postoperative pain may increase adrenergic nerve activity and plasma catecholamine concentration leading to peripheral vasoconstriction, reduced perfusion and decreased tissue oxygen partial pressure. As a consequence, peripheral tissues suffer of hypoxia that is associated with an increased incidence of surgical wound infections (Kabon et al., 2003; Waurick \& Van Aken, 2005).

Postoperative epidural analgesia substantially diminishes sympato-adrenergic response caused by nociceptive stimuli improving microcirculation and tissue oxygen partial pressure and thus might promote wound healing (Kabon et al., 2003).

Despite the improvement of postoperative immune function and tissue oxygen tension, the potential benefit of epidural analgesia on resistance to infectious complications, in particular to surgical wound infections is clinically irrelevant (Liu \& Wu, 2007).

In conclusion, there is clinical minimal evidence suggesting that epidural analgesia positively affects the risk of postoperative infections, especially wound infections in major abdominal surgery patients, as few studies have specifically addressed this question.

\subsection{Effect of epidural analgesia on cognitive function}

Given the common occurrence and its long-term effects in functioning, especially in elderly, postoperative cognitive decline is a clinically important issue in postoperative management of major abdominal surgery patients (Fong et al., 2006; Liu \& Wu, 2007; Moraca et al., 2003).

The etiology of cognitive dysfunction is still not elucidated and is probably multifactorial (Kehlet \& Holte, 2001; Liu \& Wu, 2007; Moraca et al., 2003). The cited studies do agree that preexisting patient factors, as well as intraoperative and postoperative causes are involved (Fong et al., 2006; Liu \& Wu, 2007).

Taking into account that preoperative patient-related factors are not changeable and intraoperative anesthetic types have not been shown to affect mental status, there are reports that have been focused on postoperative management strategies, the rationale for this choice being the association between postoperative pain and cognitive decline (Fong et al., 2006; Moraca et al., 2003).

As epidural analgesia provides better pain relief and spares systemic opioids, there is at least a theoretical reason for improved cognitive status with this strategy after major abdominal surgery. However, the clinical impact of postoperative epidural analgesia on cognitive dysfunction after major abdominal procedures is controversial (Liu \& Wu, 2007; Moraca et al., 2003).

There are studies that have not found any difference between epidural and parenteral analgesia with respect to postoperative cognitive decline. The small sample sizes and the 
lack of standardized approach for mental disorders monitoring could significantly limit the validity of such findings (Fong et al., 2006; Liu \& Wu, 2007).

Conversely, in other studies epidural analgesia has been shown to cause better pain control with less sedation, thus contributing to a significant improvement in postoperative cognition when compared with intravenous opioids (Kehlet \& Holte, 2001; Moraca et al., 2003).

Similar to the above-mentioned studies, these investigations are limited by small sample size, heterogeneous criteria to define postoperative cognitive dysfunction, variations in the level at which the epidural catheter is inserted, as well as the medication used, meaning local anesthetic or local anesthetic and opioid.

In conclusion, the effect of epidural analgesia on postoperative cognitive dysfunction is still unclear and the observations from this review reinforce the necessity for future investigations using appropriate and uniform outcome measures and, most importantly, enrolling a sufficient number of patients.

\subsection{Effect of epidural analgesia on postoperative mortality}

The increasing safety of current surgical procedures, together with advances in anesthetic and intensive-care practice lead to a substantial reduction in postoperative fatality, even in high-risk patients undergoing major abdominal surgery. Thus, the postoperative mortality as an end-point may not be adequate to demonstrate the benefits of epidural analgesia (Nimmo, 2004).

However, it has to be mentioned that a number of studies have investigated the effect of epidural analgesia versus standard systemic opioids on postoperative mortality in high-risk patients undergoing extensive abdominal surgery. Most of these trials have reported no significant reduction in postoperative mortality with epidural blockade (Kehlet \& Holte, 2001; Liu \& Wu, 2007; Nimmo, 2004). The criticized methodology and the small number of patients limit the interpretation of these findings.

It is of note an often-cited prospective randomized controlled study (MASTER study) that has found no difference in overall mortality between groups, but a reduced incidence of pulmonary and thromboembolic complications and significantly better analgesia in the epidural group (Liu \& Wu, 2007; Nimmo, 2004; Rigg et al., 2000; Waurick \& Van Aken, 2004). Once again this trial has been not adequately sized to assess mortality, a postoperative complication with currently low incidence and it proves insufficient evidence for valid conclusions in this respect (Liu \& Wu, 2007).

Procedure-specific meta-analyses focused on the impact of epidural analgesia on postoperative mortality have shown a benefit, but because of the occurrence of fatal events in a few studies involving a minority of the total number of subjects, the validity and clinical relevance of these results is questioned (Liu \& Wu, 2007; Nimmo, 2004).

Analysis of large-scale databases including patients undergoing a variety of extensive abdominal procedures has revealed the association of postoperative epidural analgesia with a significantly lower incidence for 7-day and 30-day mortality (Liu \& Wu, 2007). Although the number of patients is impressive this time, such analyses suffer from the retrospective 
nature and degree of association between epidural analgesia and mortality as postoperative outcome (Liu \& Wu, 2007).

In summary, our review of the literature suggests it is unlikely that any effect of epidural block translates into relevant improvement in postoperative mortality after major abdominal surgery.

\subsection{Effect of epidural analgesia on duration of intensive care unit and hospital stay}

Postoperative epidural analgesia after major abdominal procedures may induce improvements in many physiological variables, clinically translated into positive effects on paralytic ileus, pulmonary, cardiac and thromboembolic outcome. The reduction in the incidence and severity of postoperative morbidity suggests at least theoretically, a decrease in length of intensive care and hospital stay.

It is therefore surprising and somewhat disappointing that the effect of epidural analgesic techniques on duration of intensive care unit and hospital stay has been rather small or nondemonstrable by clinical trials.

Thus, large randomized studies prospectively enrolling high-risk patients undergoing major abdominal surgery have found no difference in length of stay in intensive care unit or in the hospital between subjects receiving postoperative epidural analgesia and those receiving iv opioids (Kehlet \& Holte, 2001; Peyton et al., 2003).

Other studies have noted a discrepancy between earlier achievement of intensive care discharge criteria and actual hospital stay that is not affected in patients receiving epidural analgesia compared to those treated with systemic opioids after major abdominal surgery (Kehlet \& Holte, 2001).

On the other hand, retrospective analyses have concluded that both intensive care unit and hospital length of stay have been decreased in patients treated with epidural analgesia compared to those treated with systemic opioids (Moraca et al., 2003; Rigg et al., 2002).

However, it should be emphasized that intensive care and hospital stay may be poor outcome measures since they depend on a lot of factors other than pain control and in order to demonstrate a potential reduction in their duration by epidural analgesia, this strategy has to be integrated into a multimodal rehabilitation program (Kehlet, 2008; Kehlet \& Holte, 2001).

Although there is general agreement that epidural analgesia improves postoperative outcome in major abdominal surgery patients, the progress in reducing hospital and intensive care length of stay is slow (Kehlet, 2008; Kehlet \& Wilmore, 2008).

Consequently, the well documented clinical advantages of epidural analgesia have to be used together with adjustments of postoperative care into a complex rehabilitation program to facilitate early recovery and the preliminary experience has shown a positive effect of such a collaborative efforts in reducing hospital and intensive care stay (Basse et al., 2000; Kehlet \& Holte, 2001).

\subsection{Effect of epidural analgesia on cost of postoperative care}

An economic analysis on postoperative pain treatment comparing epidural analgesia and standard intravenous opioid analgesia after major abdominal surgery becomes of interest, 
especially when health-care resources are scarce. Apart from efficacy and safety, a policy decision in selecting different analgesia strategies should take into account cost-effectiveness ratio.

Although the costs per quality adjusted life year is recommended in the available health economic literature when performing cost-effectiveness evaluation, such a measure of outcome appears inadequate for our analysis, since this review is focused on a short effect duration of acute pain treatment. Thus, the cost per gained pain-free day has been considered appropriate under these circumstances, the cited studies being in complete agreement that postoperative pain control is a mandatory component of early postoperative care (Bartha et al., 2006).

On the other hand, for a rigorous economic evaluation there are many other relevant aspects of multidimensional experience of pain, that reflect the improved health state of subject due to pain relief. Thus, other outcome measures such as length of hospital stay, morbidity and mortality should be considered (McLeod et al., 2001; Paulsen et al., 2001; Werawatganon \& Charuluxananan, 2005).

There are relatively few economic analyses of postoperative pain treatment after major abdominal procedures designed to estimate the cost-effectiveness of epidural analgesia compared with systemic opioids and the available studies have sample sizing and methodological limitations (Bartha et al., 2006). Beside all these difficulties, there is generally accepted that the cost of epidural analgesia is around three times higher than that of standard intravenous analgesia (Bartha et al., 2006). Here is a judgment of value whether this supplementary cost per pain-free day is reasonable in relation to the benefit.

Another problem to be taken into account before decision making is who will suffer because of lack of health-care (Bartha et al., 2006). Under actual circumstances of poor financial support, epidural analgesia as better choice for postoperative pain relief sometimes is not the best one. Thus, it seems reasonable to go to the second best alternative, systemic analgesia, that appears the most cost-effective analgesic modality (Bartha et al., 2006).

Before any conclusion concerning policy recommendations, such a difference in costs has to be judged closely related to parameters that have been largely ignored in previous research on health-care expenses, namely postoperative morbidity, mortality and duration of hospital stay after major abdominal surgery. This approach will help the clinical practitioner to answer the question whether epidural analgesia results in objective improvement in outcomes in major abdominal surgery patients and its related extra costs are outweighed by clinical benefits.

\section{Complications of epidural analgesia}

Epidural analgesia can be provided safely in patients undergoing major abdominal surgery since the documented risk-to-benefit ratio is convincingly in favor of this postoperative pain relief strategy (Nimmo, 2004).

Reports from centers where epidural analgesia is currently used in major abdominal surgery patients and effectively supervised by acute pain service personnel have shown a complication rate similar to that described for other forms of analgesia (McLeod et al.,2001; 
Nimmo, 2004; Rigg et al., 2002). In order to decrease the incidence of potentially severe complications, the candidates to epidural analgesia must be carefully selected. In this context, the technique should be avoided in septic subjects and those bearing coagulation disorders.

Several, less severe cardio-respiratory disturbances are straightly related to the administration of analgesic regimen into the epidural space. Thus, mild hypotension and bradycardia are well-recognized hemodynamic consequences of epidural induced sympatholysis, that could be balanced by fluid administration (Moraca et al., 2003). In patients with cardiopulmonary pathology in which postoperative fluid overload is undesirable, vasopressors represent the preferred treatment for hypotension, since both ephedrine and fluids have demonstrated comparable hemodynamic effects (Waurick \& Van Aken, 2004).

The opioid-induced respiratory dysfunction has an incidence estimated to be less than $10 \%$ comparable to systemic opioids (Moraca et al., 2003). The use of combination of local anesthetic and opioid on epidural route reduces the amount of opioid needed and thus the risk of its subsequent effect of respiratory depression (Moraca et al., 2003; Nimmo, 2004).

Systemic absorption of local anesthetic at high doses can produce seizures, coma and cardiac arrhythmias, the magnitude of such toxicity reactions being related to the type and concentration of anesthetic (Moraca et al., 2003).

Other potential problems with epidural analgesia are related to catheter placement and removal. Accidental dural puncture during needle insertion has been found in $0.16-1.3 \%$ in a series of 51.000 epidural catheters (Moraca et al., 2003).

Neurovascular damage during catheter insertion is uncommon too (Moraca et al., 2003). Thus, transient neurologic symptoms represented by sharp radicular back pain or paresthesias due to nerve root irritation by the catheter simply resolve after its removal (Moraca et al., 2003).

Injury to the spinal vasculature during catheter insertion or removal, described in approximately $3-12 \%$ of cases could rarely transform into symptomatic epidural hematoma. The incidence of epidural hematoma has been estimated to be less than 1 in 150.000 in one study, and respectively none in another series of 100.000 (Moraca et al., 2003). On the other hand, it is well demonstrated that symptomatic epidural hematomas are associated with the use of anticoagulant therapy, catheter placement or removal during anticoagulation or trauma during catheter insertion (Moraca et al., 2003).

The use of anticoagulation and the risk of epidural hematoma formation in subjects receiving epidural analgesia has been a controversial issue. Despite this debate, safety of both therapeutic anticoagulation and deep venous thrombosis prophylaxis in patients with postoperative epidural analgesia has been reported in multiple studies (Moraca et al., 2003). In any case, the risk of bleeding complications should be cautiously evaluated in balance with the potential benefits of epidural analgesia on an individual basis.

Catheter-related infections, namely meningitis and epidural abscess are rare (Moraca at al., 2003). Retaining epidural catheter in situ longer than 3 days and nonsterile technique increase the risk of these complications that lead to potentially devastating neurological disturbances. Early recognition of neurological disturbances that may herald an epidural 
hematoma or abscess completed with immediate imaging investigations and decompressive laminectomy within 8 hours of diagnosis have shown to improve neurologic prognosis (Nimmo, 2004).

Taking into account that epidural analgesia carries certain risks, despite objective improvements in outcome, in major abdominal surgery patients, vigilant monitoring during early postoperative period has to be guaranteed (Nimmo, 2004).

In other terms, modern analgesic practice inherently safe, together with professional surveillance during postoperative period could minimize the risk of potential complications, thus improving major abdominal surgery outcomes and patient satisfaction.

\section{Integration of postoperative epidural analgesia into rehabilitation program}

In recent years, extensive data have demonstrated positive physiological effects of postoperative epidural analgesia on several organ systems in major abdominal surgery patients (Kehlet, 2008). It is therefore surprising that the overall progress registered with this pain relieving technique in terms of postoperative morbidity has been limited, sometimes even non-demonstrable by clinical trials (Kehlet \& Holte, 2001). The most plausible explanation is that a single-modality intervention, namely effective pain relief, for a complex problem such as postoperative morbidity will provide few improvements in outcome (Kehlet, 2008; Kehlet \& Holte, 2001).

A multimodal approach consisting into a complex postoperative rehabilitation program seems to be more rational. The postoperative care package involves mainly four factors: effective epidural analgesia, as an essential part but not the only one, limiting the stress response to surgery, early mobilization and oral nutrition (Kehlet, 2008; Kehlet \& Wilmore, 2008; Nimmo, 2004).

The use of well-documented physiological advantages of epidural analgesia in such a postoperative care program leads to decrease of morbidity across major abdominal procedures and significantly improves the quality of postoperative recovery (Kehlet, 2008; Kehlet \& Wilmore, 2008).

Findings of many clinical trials are relevant in this respect. Thus, patients with major abdominal procedures managed in a multimodal care program including epidural analgesia have demonstrated earlier discharge from intensive-care unit, earlier return of normal bowel function, reduced catabolism and less fatigue than those undergoing equivalent surgery but not participating in such a postoperative care program (Carli et al., 2002; Kehlet, 2008; Nimmo, 2004).

A successful multimodal rehabilitation program requires the reorganization of traditional acute pain service activity, with emphasis on functional recovery (Kehlet \& Holte, 2001). Increased collaborative effort of the patient, anesthetist, surgeon and surgical nurse undoubtedly improves the rate of progress in postoperative outcome. The revision of traditional postoperative care program with drains, gastrointestinal tubes, catheters, prolonged fasting and other restrictions is mandatory, too (Kehlet \& Holte, 2001).

Educational programs have to be developed in order to analyze postoperative pathophysiology, as well as the factors that could limit early recovery after each individual major abdominal procedure (Kehlet \& Dahl, 2003; Kehlet \& Wilmore, 2008). 
A key element for multimodal rehabilitation program is the implementation and development of daily nurse care programs that could improve the quality of postoperative care, thus facilitating early recovery after major abdominal procedures (Kehlet \& Holte, 2001).

The concept of multimodal postoperative rehabilitation program with epidural analgesia as a key factor, that provides effective control of postoperative pain after major abdominal surgery allowing early mobilization and oral feeding, together with the stress-reducing effect, has gained acceptance of patients, acute pain practitioners and surgeons, being the single strategy that could significantly improve postoperative outcome.

\section{Conclusions and future directions for research}

One of the main issues of concern to patients presenting for major abdominal surgery is postoperative analgesia. Consequently, provision of high-quality postoperative pain control remains an important goal for acute pain service activity.

Epidural analgesia is considered as the gold standard analgesic technique for major abdominal procedures. This strategy has the potential to provide complete analgesia and it is particularly effective at optimizing functional pain relief, thus improving patient satisfaction and postoperative outcome.

There is consistent evidence that epidural analgesia in major abdominal surgery has positive physiological effects on cardiovascular and pulmonary functions, paralytic ileus and catabolism, all of which theoretically leading to reduced major morbidity and mortality.

The accuracy of this finding should become irrelevant because of contemporary changes in medical practice with rapid conversion of major abdominal surgery to laparoscopic procedures and implementation of fast-track programs.

A critical limitation of nearly all discussed studies is the relatively small number of patients that have been enrolled. The low incidence of postoperative complications and reduced rate of mortality require increasingly large randomized clinical trials and the acquisition of large amounts of patient data.

The concept of multimodal rehabilitation program in which epidural analgesia is a key component represents a major task. The potential positive effects of epidural analgesia on postoperative outcome might not live up to expectations unless these physiological advantages are used in the multimodal postoperative rehabilitation program. The other therapeutic factors (early mobilization, early oral nutrition, fluid balance) must be integrated into future studies examining postoperative outcome after major abdominal surgery.

With safety modern anesthetic practice and minimally invasive surgical techniques it is the time to increase the scope of definition of outcomes of interest. As mortality and major morbidity become uncommon, even in high-risk abdominal procedures, the future studies may need to focus on patient's own views of recovery. These nontraditional outcomes assessed from the patient's perspective comprise quality of postoperative recovery including analgesia, well being state, return to preoperative energy and activity level and quality of life.

Epidural analgesia is time-consuming, it requires specific technical skills and pharmacological abilities and professional surveillance. Clearly, epidural analgesia is not devoid of risks and failures may occur. 
Our analysis provide an evidence base for rational decision making to help the anesthesiologist in choosing the most beneficial use of epidurals in major abdominal surgery patients.

\section{References}

Agnelli, G. (2004). Prevention of venous thromboembolism in surgical patients. Circulation, Vol. 110, No. 24, Suppl. IV, (December 2004), pp. 4-12, ISSN: 1524-4539

Ballantyne, J.; Carr, D. \& deFerranti, S. (1998). The comparative effects of postoperative analgesic therapies on pulmonary outcome: Cumulative meta-analyses of randomized, controlled trials. Anesthesia \& Analgesia, Vol. 86, No. 3, (March 1998), pp. 598-612, ISSN 1526-7598

Bartha, E.; Carlsson, P. \& Kalman, S. (2006). Evaluation of costs and effects of epidural analgesia and patient-controlled intravenous analgesia after major abdominal surgery. British Journal of Anaesthesia, Vol. 96, No. 1, (January 2006), pp. 111-117, ISSN 1471-6771

Basse, L.; Hjort, J. \& Billesbølle, P. (2000). A clinical pathway to accelerate recovery after colonic resection. Annals of Surgery, Vol. 232, No. 1, (July 2000), pp. 51-57, ISSN 1528-1140

Bauer, A. \& Boeckxstaens, G. (2004). Mechanisms of postoperative ileus. Neurogastroenterology $\mathcal{E}$ Motility, Vol. 16, Issue Supplement s2, (October 2004), pp. 54-60

Beattie, S.; Badner, N. \& Choi, P. (2001). Epidural analgesia reduces postoperative myocardial infarction: A meta-analysis. Anesthesia \& Analgesia, Vol. 93, No. 4, (October 2001), pp. 853-858, ISSN 1526-7598

Beilin, B.; Shavit, Y. \& Trabekin, E. (2003). The effects of postoperative pain management on immune response to surgery. Anesthesia \& Analgesia, Vol. 97, No. 3, (September 2003), pp. 822-827, ISSN 1526-7598

Block, B.; Liu, S. \& Rowlingson, A. (2003). Efficacy of postoperative epidural analgesia: A meta-analysis. The Journal of the American Medical Association, Vol. 290, No. 18, (November 2003), pp. 2455-2463, ISSN: 1538-3598

Bombeli, T. \& Spahn, D. (2004). Updates in perioperative coagulation: Physiology and management of thromboembolism and haemorrhage. British Journal of Anaesthesia, Vol. 93, No. 2, (August 2004), pp. 275-287, ISSN 1471-6771

Carli, F.; Mayo, N. \& Klubien, K. (2002). Epidural analgesia enhances functional exercise capacity and health-related quality of life after colonic surgery: results of a randomized trial. Anesthesiology, Vol.97, No. 3, (September 2002), pp. 540-549, ISSN 0003-3022

Devereaux, P.; Goldman, L. \& Cook, D. (2005). Perioperative cardiac events in patients undergoing noncardiac surgery: A review of the magnitude of the problem, the pathophysiology of the events and methods to estimate and communicate risk. Canadian Medical Association Journal, Vol. 173, No. 6, (September 2005), pp. 627-634, ISSN 1488-2329

Dolin, S.; Cashman, J. \& Bland, J. (2002). Effectiveness of acute postoperative pain management: I. Evidence from published data. British Journal of Anaesthesia, Vol. 89, No. 3, (September 2002), pp. 409-423, ISSN 1471-6771

Fong, H.; Sands, L. \& Leung, J. (2006). The role of postoperative analgesia in delirium and cognitive decline in elderly patients: A systematic review. Anesthesia $\mathcal{E}$ Analgesia, Vol. 102, No. 4, (April 2006), pp. 1255-1266, ISSN 1526-7598

Grass, J. (2000). The role of epidural anesthesia and analgesia in postoperative outcome. Anesthesiology Clinics of North America, Vol. 18, No. 2, (June 2000), pp. 407-428 
Groeben, H.; Schäfer, B. \& Pavlakovic, G. (2002). Lung function under high thoracic segmental epidural anesthesia with ropivacaine or bupivacaine in patients with severe obstructive pulmonary disease undergoing breast surgery. Anesthesiology, Vol. 96, No. 3, (March 2002), pp. 536-541, ISSN 0003-3022

Gruber, E.; Tschernko, E. \& Kritzinger, M. (2001). The effects of thoracic epidural analgesia with bupivacaine $0.25 \%$ on ventilatory mechanics in patients with severe chronic obstructive pulmonary disease. Anesthesia \& Analgesia, Vol. 92, No. 4, (April 2001), pp. 1015-1019, ISSN 1526-7598

Holte, K. \& Kehlet, H. (2002). Epidural anaesthesia and analgesia - effects on surgical stress responses and implications for postoperative nutrition. Clinical Nutrition, Vol. 21, No. 3, (June 2002), pp. 199-206, ISSN 0261-5614

Jiang, S.; Li, Z. \& Huang, L. (2005). Multivariate analysis of the risk for pulmonary complication after gastrointestinal surgery. World Journal of Gastroenterology, Vol. 11, No. 24, (June 2005), pp. 3735-3741, ISSN 1007-9327

Jørgensen, H.; Wetterslev, J. \& Møiniche, S. (2000). Epidural local anaesthetics versus opioidbased analgesic regimens on postoperative gastrointestinal paralysis, PONV and pain after abdominal surgery. In: Cochrane Database of Systematic Reviews 2001, 23.10.2000, Available from http://www2.cochrane.org/reviews/en/ab001893.html

Kabon, B.; Fleischmann, E. \& Treschan, T. (2003). Thoracic epidural anesthesia increases tissue oxygenation during major abdominal surgery. Anesthesia $\mathcal{E}$ Analgesia, Vol. 97, No. 6, (December 2003), pp. 1812-1816, ISSN 1526-7598

Kehlet, H. (1994). Postoperative pain relief-what is the issue? British Journal of Anaesthesia, Vol. 72, No. 4, (April 1994), pp. 375-378, ISSN 1471-6771

Kehlet, H. (1997). Multimodal approach to control postoperative pathophysiology and rehabilitation. British Journal of Anaesthesia, Vol. 78, No. 5, (May 1997), pp. 606-617, ISSN 1471-6771

Kehlet, H. \& Holte, K. (2001). Effect of postoperative analgesia on surgical outcome. British Journal of Anaesthesia, Vol. 87, No. 1, (June 2001), pp. 62-72, ISSN 1471-6771

Kehlet, H. \& Dahl, J. (2003). Anaesthesia, surgery and challenges in postoperative recovery. The Lancet, Vol. 362, No. 9399, (December 2003), pp. 1921-1928

Kehlet, H. (2008). Fast-track colorectal surgery. The Lancet, Vol. 371, No. 9615, (March 2008), pp. 791-793

Kehlet, H. \& Wilmore, D. (2008). Evidence-based surgical care and the evolution of fast-track surgery. Annals of Surgery, Vol. 248, No. 2, (August 2008), pp. 189-198, ISSN 1528-1140

$\mathrm{Liu}$, S. \& Wu, C. (2007). Effect of postoperative analgesia on major postoperative complications: A systemic update of the evidence. Anesthesia $\mathcal{E}$ Analgesia, Vol. 104, No. 3, (March 2007), pp. 689-702, ISSN 1526-7598

Nigärd, E.; Kofoed, K. \& Freiberg, J. (2005). Effects of high thoracic epidural analgesia on myocardial blood flow in patients with ischemic heart disease. Circulation, Vol. 111, No. 17, (May 2005), pp. 2165-2170, ISSN: 1524-4539

Nimmo, S. (2004). Benefit and outcome after epidural analgesia. Continuing Education in Anaesthesia, Critical Care \& Pain, Vol. 4, No. 2, (April 2004), pp. 44-47, ISSN 1743-1824

McLeod, G.; Davies, H. \& Munnoch N. (2001). Postoperative pain relief using thoracic epidural analgesia: Outstanding success and disappointing failures. Anaesthesia, Vol. 56, No. 1, (January 2001), pp. 75-81

Moraca, R.; Sheldon, D. \& Thirlby, R. (2003). The role of epidural anesthesia and analgesia in surgical practice. Annals of Surgery, Vol. 238, No. 5, (November 2003), pp. 663-673, ISSN 1528-1140 
Mythen, M. (2005). Postoperative gastrointestinal tract dysfunction. Anesthesia $\mathcal{E}$ Analgesia, Vol. 100, No. 1, (January 2005), pp. 196-204, ISSN 1526-7598

Park, W.; Thompson, J. \& Lee, K. (2001). Effect of epidural anesthesia and analgesia on perioperative outcome: A randomized, controlled veterans affairs cooperative study. Annals of Surgery, Vol. 234, No. 4, (October 2001), pp. 560-571, ISSN 1528-1140

Paulsen, E.; Porter, M. \& Helmer, S. (2001). Thoracic epidural versus patient-controlled analgesia in elective bowel resections. The American Journal of Surgery, Vol. 182, No. 6, (December 2001), pp. 570-577, ISSN 0002-9610

Peyton, P.; Myles, P. \& Silbert, B. (2003). Perioperative epidural analgesia and outcome after major abdominal surgery in high-risk patients. Anesthesia $\mathcal{E}$ Analgesia, Vol. 96, No. 2, (February 2003), pp. 548-554, ISSN 1526-7598

Pöpping, D.; Elia, N. \& Marret, E. (2008). Protective effects of epidural analgesia on pulmonary complications after abdominal and thoracic surgery. Archives of Surgery, Vol. 143, No. 10, (October 2008), pp. 990-999

Rigg, J.; Jamrozik, K. \& Myles, P. (2000). Design of the multicenter Australian study of epidural anesthesia and analgesia in major surgery: The MASTER trial. Controlled Clinical Trials, Vol. 21, No. 3, (June 2000), pp. 244-256

Rigg, J.; Jamrozik, K. \& Myles, P. (2002). Epidural anaesthesia and analgesia an outcome of major surgery: A randomized trial. The Lancet, Vol. 359, No. 9314, (April 2002), pp. 1276-1282

Roderick, P.; Ferris, G. \& Wilson, K. (2005). Towards evidence-based guidelines for the prevention of venous thromboembolism: Systematic reviews of mechanical methods, oral anticoagulation, dextran and regional anaesthesia as thromboprophylaxis. Health Technology Assessment, Vol. 9, No. 49, (December 2005), pp. 1-94

Sido, B.; Teklote, J. \& Hartel, M. (2004). Inflammatory response after abdominal surgery. Best Practice \& Research: Clinical Anaesthesiology, Vol. 18, No. 3, (September 2004), pp. 439-454, ISSN 1521-6896

Warner, D. (2000). Preventing postoperative pulmonary complications: the role of anesthesiologist. Anesthesiology, Vol. 92, No. 5, (May 2000), pp.1467-1472, ISSN 0003-3022

Waurick, R. \& Van Aken, H. (2005). Update in thoracic epidural anaesthesia. Best Practice $\mathcal{E}$ Research: Clinical Anaesthesiology, Vol. 19, No. 2, (June 2005), pp.201-213, ISSN 1521-6896

Werawatganon, T. \& Charuluxananan, S. (2005). Patient controlled intravenous opioid analgesia versus continuous epidural analgesia for pain after intra-abdominal surgery. Anesthesia \& Analgesia, Vol. 100, No. 5, (May 2005), pp. 1536, ISSN 1526-7598

Wheatley, R.; Shug, S. \& Watson, D. (2001). Safety and efficacy of postoperative epidural analgesia. British Journal of Anaesthesia, Vol. 87, No. 1, (June 2001), pp. 47-61, ISSN 1471-6771

Wu, C.; Cohen, S. \& Richman, J. (2005). Efficacy of postoperative patient-controlled and continuous infusion epidural analgesia versus intravenous patient-controlled analgesia with opioids: A meta-analysis. Anesthesiology, Vol. 103, No. 5, (November 2005), pp. 1079-1088, ISSN 0003-3022

Yokoyama, M.; Itano, Y. \& Katayama, H. (2005). The effects of continuous epidural anesthesia and analgesia on stress response and immune function in patients undergoing radical esophagectomy. Anesthesia \& Analgesia, Vol. 101, No. 5, (November 2005), pp. 1521-1527, ISSN 1526-7598 


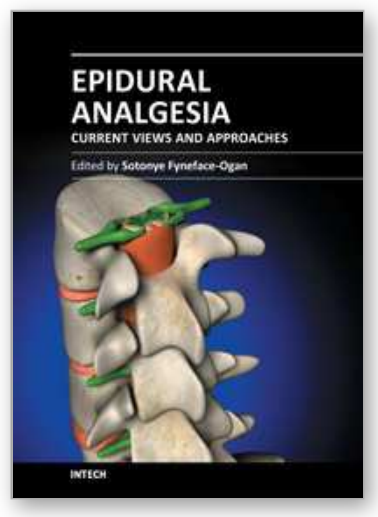

\author{
Epidural Analgesia - Current Views and Approaches \\ Edited by Dr. Sotonye Fyneface-Ogan
}

ISBN 978-953-51-0332-5

Hard cover, 174 pages

Publisher InTech

Published online 16, March, 2012

Published in print edition March, 2012

Epidural analgesia is a form of pain relief administered through the space surrounding the dural sheath either by direct injection or via catheter. The agent, when administered, can cause both a loss of sensation (anesthesia) and a loss of pain (analgesia), by reversibly interrupting the transmission of signals through nerves in or near the spinal cord. This form of pain relief has been found useful in many clinical situations. This book intends to provide an in-depth review of the current knowledge on epidural analgesia. The use of this form of analgesia is explored by contributors from different perspectives, including labor and delivery, postoperative analgesia in both pediatric and geriatric patients, and its role during anesthesia and surgery. In order to provide a balanced medical view this book was edited by an obstetric anesthesiologist.

\title{
How to reference
}

In order to correctly reference this scholarly work, feel free to copy and paste the following:

Iulia Cindea, Alina Balcan, Viorel Gherghina, Bianca Samoila, Dan Costea, Catalin Grasa and Gheorghe Nicolae (2012). The Impact of Epidural Analgesia on Postoperative Outcome After Major Abdominal Surgery, Epidural Analgesia - Current Views and Approaches, Dr. Sotonye Fyneface-Ogan (Ed.), ISBN: 978-953-510332-5, InTech, Available from: http://www.intechopen.com/books/epidural-analgesia-current-views-andapproaches/the-impact-of-epidural-analgesia-on-postoperative-outcome-after-major-abdominal-surgery

\section{INTECH}

open science | open minds

\author{
InTech Europe \\ University Campus STeP Ri \\ Slavka Krautzeka 83/A \\ 51000 Rijeka, Croatia \\ Phone: +385 (51) 770447 \\ Fax: +385 (51) 686166 \\ www.intechopen.com
}

\author{
InTech China \\ Unit 405, Office Block, Hotel Equatorial Shanghai \\ No.65, Yan An Road (West), Shanghai, 200040, China \\ 中国上海市延安西路65号上海国际贵都大饭店办公楼 405 单元 \\ Phone: +86-21-62489820 \\ Fax: +86-21-62489821
}


(C) 2012 The Author(s). Licensee IntechOpen. This is an open access article distributed under the terms of the Creative Commons Attribution 3.0 License, which permits unrestricted use, distribution, and reproduction in any medium, provided the original work is properly cited. 